\title{
Effects of oral phosphocysteamine and rectal cysteamine in cystinosis
}

\author{
W G van't Hoff, T Baker, R N Dalton, L C Duke, S P Smith, C Chantler, G B Haycock
}

\begin{abstract}
Diurnal variation in leucocyte cystine and the effects of equimolar single doses of oral phosphocysteamine and rectal cysteamine were studied in eight patients with cystinosis, aged 1.8-16.5 years. No significant diurnal variation in leucocyte cystine was found. Absorption of cysteamine was reduced after rectal administration compared with the oral dose: mean (SD) peak concentration $17 \cdot 2(6 \cdot 3) \mu \mathrm{mol} / 1 \mathrm{v}$ $36.4(5.5) \mu \mathrm{mol} / \mathrm{l}$ at $40 \mathrm{~min}$ and mean (SD) area under the curve $22.3(14 \cdot 3) v 59.4(33.1) \mu \mathrm{mol} /$ h/l. Oral phosphocysteamine significantly reduced the mean (SD) leucocyte cystine from $8.09(0.47)$ to $3.26(1.48) \mathrm{nmol} 1 / 2$ cystine/ $\mathrm{mg}$ protein at three hours. At 12 hours the mean leucocyte cystine was significantly lower than the pretreatment concentration. Rectal cysteamine did not significantly reduce the mean leucocyte cystine concentration. In conclusion, phosphocysteamine suspension may be administered every 12 hours. Rectal cysteamine administration is feasible but higher doses are required before efficacy can be judged.
\end{abstract}

Nephropathic cystinosis is a rare autosomal recessive condition in which the amino acid cystine accumulates in the lysosomes. ${ }^{1}$ Affected children usually present in infancy with impaired growth and features of Fanconi's syndrome. ${ }^{1}$ Progressive renal damage occurs and, without renal transplantation, most children die in the first decade. ${ }^{1}$ Other tissues are damaged by the progressive cystine accumulation so that the older transplanted patients can develop hypothyroidism, retinopathy, myopathy, pancreatic dysfunction, and dementia. ${ }^{2-4}$

The biochemical abnormality in cystinosis is a defect in the lysosomal transport of cystine, which leads to excessive cystine accumulation within the lysosomes. ${ }^{1}$ This can be determined biochemically in a preparation of leucocytes from a patient. Cysteamine has been shown to reduce the cystine accumulation both in vitro and in vivo. ${ }^{5}$ It acts by undergoing disulphide exchange with cystine to form the cysteinecysteamine mixed disulphide, which is transported out of the lysosome. ${ }^{6}$ Long term cysteamine treatment in patients with cystinosis has been shown to reduce the rate of progression of renal damage and improve growth. ${ }^{7}$ To overcome the unpleasant taste and smell of cysteamine the prodrug, phosphocyteamine, was developed. ${ }^{8}$ Phosphocysteamine is rapidly hydrolysed in the stomach to cysteamine, the active form. ${ }^{9}$ Despite their use for over 12 years the pharmacokinetics, optimal route, and frequency of administration of these drugs have yet to be determined. We have undertaken single dose studies of the pharmacokinetics and pharmacodynamics of oral phosphocysteamine and rectal cysteamine.

\section{Patients and methods}

PATIENTS

Eight patients with cystinosis were studied. Their details are given in table 1. Renal function was stable at the time of study and none had biochemical evidence of liver disease (plasma bilirubin concentration and aspartate transaminase and alkaline phosphatase activities were within normal limits). Informed, written consent was obtained from the parents and from the older children.

\section{STUDY PROTOCOLS}

Patients who were already on cysteamine or phosphocysteamine treatment stopped the drug seven days before the study began to allow an adequate 'wash-out' period. Phosphocysteamine was chosen for the oral dose study because most patients find it more acceptable than cysteamine.
Guy's Hospital, St Thomas' Street, London SE1 9RT, Evelina Children's Department W G van't Hoff T Baker

R N Dalton

C Chantler

G B Haycock

Department of Pharmacy

L C Duke

S P Smith

Correspondence to:

Dr van't Hoff.

Accepted 25 July 1991

\section{Table 1 Patient details}

\begin{tabular}{|c|c|c|c|c|c|}
\hline $\begin{array}{l}\text { Patient } \\
\text { No }\end{array}$ & $\begin{array}{l}\text { Age } \\
\text { (years) }\end{array}$ & Sex & $\begin{array}{l}\text { Renal } \\
\text { transplant }\end{array}$ & $\begin{array}{l}\text { Plasma } \\
\text { creatinine } \\
(\mu \text { molll })\end{array}$ & $\begin{array}{l}\text { Other } \\
\text { medication }\end{array}$ \\
\hline $\begin{array}{l}1 \\
2 \\
3 \\
4 \\
5\end{array}$ & $\begin{array}{r}1 \cdot 8 \\
2 \cdot 0 \\
4 \cdot 5 \\
10 \cdot 2 \\
10 \cdot 6\end{array}$ & $\begin{array}{l}\mathbf{M} \\
\mathbf{F} \\
\mathbf{F} \\
\mathbf{M} \\
\mathbf{M}\end{array}$ & $\begin{array}{l}\text { No } \\
\text { No } \\
\text { No } \\
\text { No } \\
\text { Yes }\end{array}$ & $\begin{array}{r}79 \\
40 \\
79 \\
186 \\
68\end{array}$ & $\begin{array}{l}\text { Cyproheptadine } \\
\text { None } \\
\text { Indomethacin } \\
\text { None } \\
\text { Prednisolone, cyclosporin, }\end{array}$ \\
\hline $\begin{array}{l}6 \\
7\end{array}$ & $\begin{array}{l}13 \cdot 9 \\
16 \cdot 1\end{array}$ & $\stackrel{\mathbf{M}}{\mathbf{F}}$ & $\begin{array}{l}\text { Yes } \\
\text { Yes }\end{array}$ & $\begin{array}{l}54 \\
59\end{array}$ & $\begin{array}{l}\text { Prednisolone, azathioprine } \\
\text { Prednisolone, azathioprine, } \\
\text { trimethoprim }\end{array}$ \\
\hline 8 & 16.5 & $\mathbf{F}$ & Yes & 192 & $\begin{array}{l}\text { Prednisolone, cyclosporin, } \\
\text { azathioprine, atenolol, } \\
\text { frusemide }\end{array}$ \\
\hline
\end{tabular}


As palatability was not a concern for the rectal study, and there is no information on rectal hydrolysis of phosphocysteamine, cysteamine was used for this route. Other drug treatment was not altered during the study periods because of the hazards of withdrawing indomethacin in the pretransplant group and immunosuppression in the transplanted children. Food was withheld for eight hours before and for the first three hours of each study but free access to clear fluids was allowed in view of their obligatory polyuria.

In order to study diurnal variation in leucocyte cystine, six patients (numbers 3-8) first had serial blood samples taken for leucocyte cystine determination over a 24 hour period (at 0800 , $0900,1100,1400,2000$, and 0800). Then patients $2-8$ received a $23 \mathrm{mg} / \mathrm{kg}$ single oral dose of phosphocysteamine, given as a suspension in $50 \mathrm{ml}$ water in the morning (0730-0830). Because of the unpalatability of the drug $50 \mathrm{ml}$ of either juice or milk were allowed immediately after the drug. Serial blood samples were taken from an indwelling intravenous cannula for measurement of plasma cysteamine concentration $(1 \mathrm{ml})$ at $0,20,30,40 \mathrm{~min}, 1,1 \cdot 5,2,3$, $4,6,12$ hours and for leucocyte cystine determination $(3 \mathrm{ml})$ at $0,1,3,6$, and 12 hours after the dose. Intake of diet, fluids, and other drugs was similar on both the predose (diurnal variation) and oral dose day.

In a separate study six patients (numbers $1-6)$, received an equimolar dose $(10 \mathrm{mg} / \mathrm{kg})$ of cysteamine, given rectally as a gel. The dose of cysteamine base for each patient was dissolved in $9 \%$ methylcellulose gel to a final volume of between 2.5 and $5 \mathrm{ml}$ so that the dose volume was approximately similar for all patients. The gel was tested for stability and kept at $4^{\circ} \mathrm{C}$. Before use, it was allowed to stand at room temperature for $30 \mathrm{~min}$. Gel was administered to the patients in either the left lateral or supine position, over two minutes, using a $5 \mathrm{ml}$ syringe (Steriseal) and a fine soft plastic quill (Avon Medicals). The children remained supine for 15 min after the dose. Blood sampling times were the same as in the oral dose study.

The three younger patients (numbers 1-3) who were experiencing severe vomiting and anorexia from oral cysteamine or phosphocysteamine, were offered a trial of regular rectal cysteamine. The starting dose of $10 \mathrm{mg} / \mathrm{kg}$ cysteamine once a day was increased as tolerated. Leucocyte cystine concentrations (12 hours after the last dose) were determined at each clinic attendance. Their parents were taught how to administer each dose and kept a diary to monitor adverse reactions. Patients 1 and 2 were also started on oral indomethacin to control polyuria. ${ }^{10}$

The study protocols were approved by the Guy's Hospital ethical review committee.

\section{ANALYTICAL METHODS}

Plasma cysteamine was assayed using a modification of the method of Smolin and Schneider. ${ }^{11}$ Plasma was separated immediately and stored at $-70^{\circ} \mathrm{C}$ pending analysis. The samples were derivatised using sodium borohydride to reduce all the disulphides. After protein precipitation, cysteamine was separated by high performance liquid chromatography and measured using electrochemical detection. The limit of detection was $1 \mu \mathrm{mol} / \mathrm{l}$ and the coefficients of variation were $12 \cdot 0,4 \cdot 7$, and $4 \cdot 7 \%$ at 5,20 , and 100 $\mu \mathrm{mol} / 1$ respectively.

Leucocyte cystine concentration was determined by the method of Greene et al. ${ }^{12}$ The result was factored by the protein content of the leucocyte pellet which was measured by a modification of the Lowry method. ${ }^{13}{ }^{14}$ In our laboratory, the limit of detection of the cystine assay is $0.05 \mathrm{nmol} 1 / 2$ cystine/mg protein and the coefficients of variation are $24.7,6.0$ and $15 \cdot 2 \%$ at $0 \cdot 06,3 \cdot 54$, and $8 \cdot 47 \mathrm{nmol} 1 / 2$ cystine $/ \mathrm{mg}$ protein respectively. Plasma creatinine was measured using the standard Jaffé reaction.

\section{PHARMACOKINETIC AND STATISTICAL METHODS} Pharmacokinetic parameters were estimated from standard formulae using the BIOPAK software package. For each plasma cysteamine profile the maximum cysteamine concentration $\left(C_{\max }\right)$, and time at which this occurred $\left(\mathrm{T}_{\max }\right)$, were noted. The area under the plasma concentration time curve $\left(\mathrm{AUC}_{0-6} \mathrm{~h}\right)$ was determined by the linear trapezoidal method. Individual semilog plots of plasma concentration versus time were inspected to determine the appropriate data points from which to calculate the elimination half life $\left(t^{1 / 2} \beta\right)$. Statistical analyses were performed using a two tailed Student's paired $t$ test with Bonferroni's correction for multiple analyses. Significance was assumed if $p<0.05$.

\section{Results}

All the patients in the oral dose study successfully swallowed the phosphocysteamine suspension. Two patients (4 and 8 ) suffered vomiting, starting approximately one hour after the dose. There were no other adverse effects. Administration of the rectal cysteamine gel was well tolerated in the younger three children. The older three children who received rectal gel (4-6) expressed a desire to defaecate approximately $15 \mathrm{~min}$ after administration, but were able to retain the dose for a further $15 \mathrm{~min}$. No child in the rectal study suffered vomiting, diarrhoea, or rectal bleeding. The smell of cysteamine on the breath was very noticeable after oral dosage but minimal or not apparent after rectal dosage.

The mean leucocyte cystine concentrations for the six patients in whom diurnal variation was studied are shown in fig 1 . There was a trend for the leucocyte cystine to fall during the morning but there was no significant change from the baseline concentration at zero time ( $>0.3$ at all time points). Inspection of diet and drug sheets revealed no association between intake and leucocyte cystine.

The mean leucocyte cystine concentrations after oral and rectal doses are shown in fig $2 \mathrm{~A}$. After the oral dose, the mean leucocyte cystine concentration was significantly reduced $(p=0.05)$ to a minimum of $40 \%$ of the pretreatment concentration at three hours. At 12 hours 


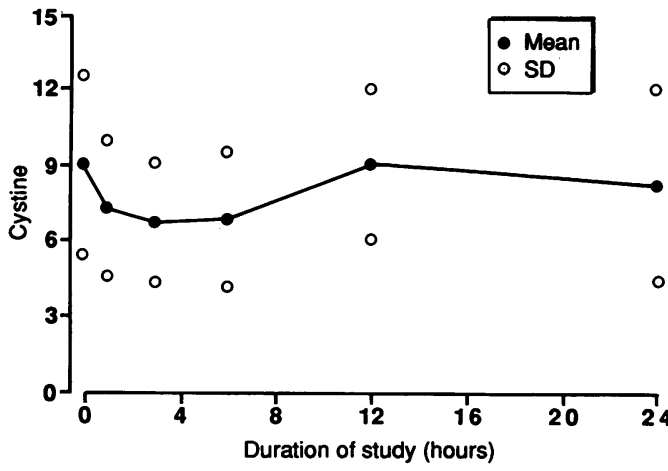

Figure 1 Diurnal variation in leucocyte cystine concentration (nmol 1/2 cystine/mg protein). Studies were started between 0730 and 0830 .
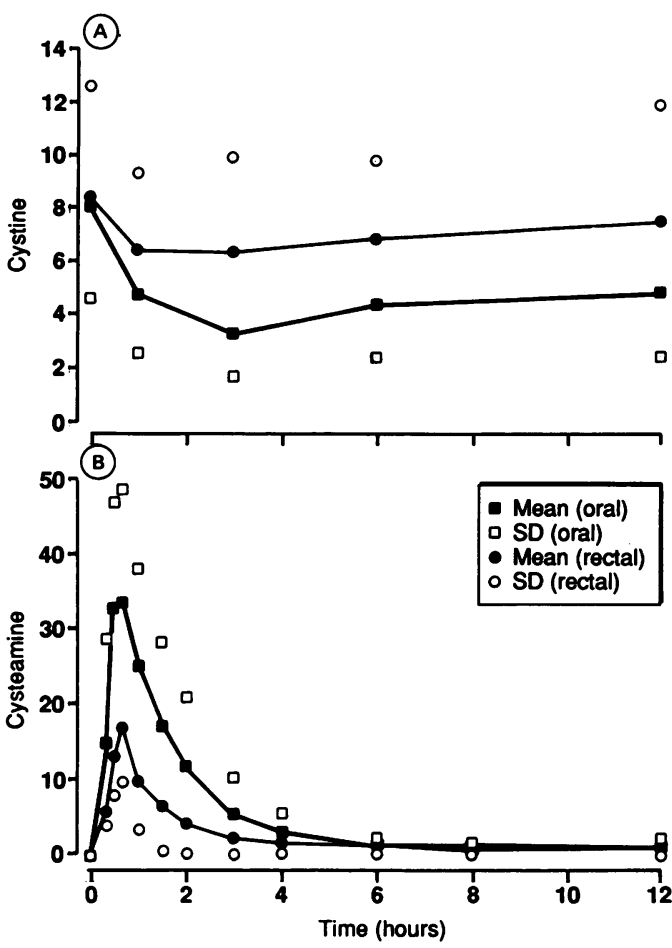

Figure 2 (A) Leucocyte cystine (nmol 1/2 cystine/mg protein) and $(B)$ plasma cysteamine $(\mu \mathrm{mol} / \mathrm{l})$ after oral phosphocysteamine and rectal cysteamine.

after the dose the mean leucocyte cystine had only reaccumulated to $61 \%$ of the pretreatment concentration $(p=0.032)$. There was no significant reduction in mean leucocyte cystine after rectal cysteamine ( $p>0.1$ at all time points).

The mean plasma cysteamine profiles after oral and rectal dosage are shown in fig $2 \mathrm{~B}$, and the derived pharmacokinetic paremeters are given in table 2 . Inspection of the plasma cysteamine profile for patient 1 in the rectal study showed a slight rise in cysteamine concentration at eight hours, making calculation of pharmacokinetic parameters impossible. The time to peak concentration $\left(T_{\max }\right)$ was the same in both studies but the mean $\mathrm{C}_{\max }$ and AUC were significantly reduced after rectal dosage ( $p=0.02$ and $p=0.03$, respectively). There was no significant difference between the mean elimination half lives, $t^{1 / 2} \beta$, in the oral and rectal dose studies $(\mathrm{p}>0 \cdot 2)$.

The parents of the children receiving regular rectal cysteamine found no difficulties in admin-
Table 2 Pharmacokinetic parameters after oral administration of phosphocysteamine and rectal administration of cysteamine. Values are mean $(S D)$

\begin{tabular}{lllll}
\hline Route & $\begin{array}{l}C_{\max } \\
(\mu \mathrm{mol} / \mathrm{l})\end{array}$ & $\begin{array}{l}T_{\max } \\
(\min )\end{array}$ & $\begin{array}{l}\text { AUC } \\
(\mu \mathrm{mol} / \mathrm{h} / \mathrm{l})\end{array}$ & $\begin{array}{l}\text { Half life } \\
(\text { hours })\end{array}$ \\
\hline Oral & $36 \cdot 4(15 \cdot 5)^{*}$ & 40 & $59 \cdot 4(33 \cdot 1)^{* * *}$ & $1 \cdot 59(1 \cdot 48) \dagger$ \\
Rectal & $17 \cdot 2(6 \cdot 3)^{*}$ & 40 & $22 \cdot 3(14 \cdot 3)^{* * *}$ & $0 \cdot 78(0 \cdot 46) \dagger$ \\
\hline
\end{tabular}

$\mathrm{C}_{\max }$ and $\mathrm{T}_{\max }$ are maximum cysteamine concentration and the time it occurred. AUC is area under the plasma concentration

${ }^{*} \mathrm{p}=0.02,{ }^{* *} \mathrm{p}=0.03, \mathrm{tp}>0.2$.

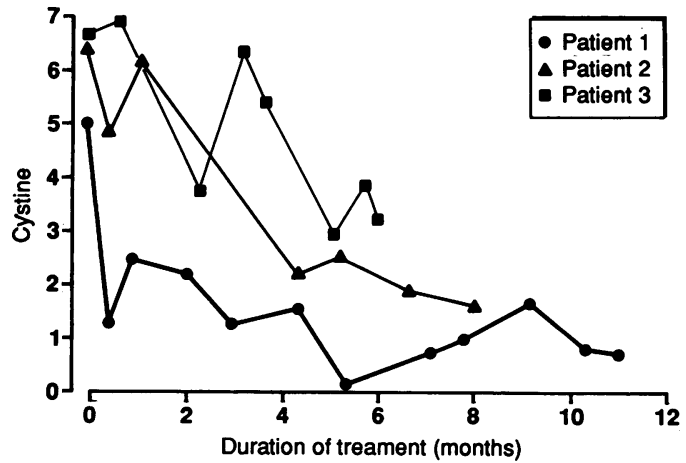

Figure 3 Leucocyte cystine (nmol $1 / 2$ cystine/mg protein) before treatment (shown at zero), and after treatment with rectal cysteamine. The pretreatment value in patient 1 was obtained before any cysteamine treatment. The pretreatment values in patients 2 and 3 are the means of three and four determinations respectively, taken while on oral

phosphocysteamine.

istering the doses whereas all three children had refused oral cysteamine or phosphocysteamine (and had also refused other oral medication). Their leucocyte cystine concentrations are shown in fig 3. The adverse reaction diaries showed that the children tended to defaecate 30 min after the dose in approximately $30 \%$ of administrations. Their parents noted that halitosis and vomiting after the cysteamine was much reduced. Two of these three patients have now stopped rectal cysteamine and recommended oral phosphocysteamine (one on account of diarrhoea and the other after an episode of minor rectal bleeding).

\section{Discussion}

Cysteamine and phosphocysteamine are the only specific drugs available to reduce cystine accumulation in cystinosis. Treatment is started as soon as the diagnosis is made (usually around 1-2 years of age) and continued until end stage renal failure supervenes. We believe that in view of the multisystem complications, there are significantly strong theoretical grounds to continue treatment after renal transplantation. Thus treatment is effectively for life.

Since the accumulation of cystine within the lysosomes is derived from protein catabolism, ${ }^{15}$ it might be expected that leucocyte cystine concentration would vary with diet and time of day. Our results show that there is no significant diurnal variation in leucocyte cystine concentrations.

After oral administration of a suspension of phosphocysteamine, cysteamine was rapidly 
absorbed. Pharmacokinetic parameters for cysteamine have not previously been described. In our study there was considerable individual variation in $C_{\max }$, AUC, and $t^{1 / 2} \beta$, but in all cases plasma cysteamine was virtually undetectable after six hours. Our plasma cysteamine concentrations are in the same range as those determined by Jonas and Schneider who concluded that the drug should be given in divided daily dosage. ${ }^{16}$ Subsequently, cysteamine and phosphocysteamine have been administered every six hours. Smolin et al measured the effects of cysteamine and phosphocysteamine on leucocyte cystine for up to six hours and found them to be equally effective. ${ }^{9}$ Our results demonstrate that the effect of the drug is more prolonged as the mean leucocyte cystine concentration had risen to only $61 \%$ of the pretreatment concentration at 12 hours after the oral dose. A study is planned to confirm whether this prolonged effect of the drug occurs after multiple dosing and with phosphocysteamine capsules instead of suspension. We now advise patients to take phosphocysteamine every 12 hours and we monitor their leucocyte cystine concentrations before a morning dose.

Cysteamine had an unpleasant taste, and after an oral dose the patient's breath smells of free sulphides. A second common side effect is vomiting, typically occurring half an hour or more after the dose. In an attempt to circumvent these problems, we investigated whether rectal administration of cysteamine was feasible and efficacious. The three younger patients receiving rectal cysteamine tolerated the dose without any problems. There was appreciable individual variation in the plasma cysteamine and leucocyte cystine profiles after rectal dosage. Although cysteamine was rapidly absorbed from the rectum, the extent of absorption was reduced when compared with the oral route (table 2). There was no significant effect on the mean leucocyte cystine concentrations (see fig 2A) and this is probably because the older three patients expelled the dose $30 \mathrm{~min}$ after administration. Halitosis was noted in two of the patients who had received rectal cysteamine but was minimal compared with that after oral dosage.

The parents of the three patients who received regular rectal cysteamine felt that it was more acceptable than the oral preparation. Compliance improved not only for cysteamine but also for their electrolyte replacements and dietary intake. Their leucocyte cystine concentrations fell towards the accepted therapeutic range $(<1$ nmol $1 / 2$ cystine/mg protein; see fig 3 ). Patient 1 had low cystine concentrations on a comparatively low final dose of cysteamine $(20 \mathrm{mg} / \mathrm{kg}$ ) day) whereas the other two patients required doses similar to those used in oral treatment $(70$ and $50 \mathrm{mg} / \mathrm{kg} /$ day, respectively). For two of the three patients, rectal cysteamine was a 'stopgap' treatment, covering a period in which they were completely intolerant of the oral medication. They are now re-established on oral phosphocysteamine. The short duration of rectal treatment and the small numbers of patients treated preclude any analysis of its effect on renal function.

The results in the present study suggest that, when given as an oral suspension, phosphocysteamine may be administered every 12 hours. Such a reduction in the frequency of administration is of enormous practical benefit to the patients. Rectal administration proved to be feasible and safe. For some children unable to tolerate oral cysteamine or phosphocysteamine, rectal administration may be an alternative.

We are grateful to Children Nationwide Medical Research who supported WG van't Hoff, RN Dalton, and the Childre Nationwide Kidney Research Laboratory at Guy's Hospital. T Baker was supported by the National Kidney Research Fund.

1 Gahl WA, Renlund M, Thoene JG. Lysosomal transpor disorders: cystinosis and sialic acid storage disorders. In Scriver CR, Beaudet AL, Sly WS, Valle D. eds. The metabolic basis of inherited disease. New York: McGraw-Hill, 1989:2619-35.

2 Gahl WA, Kaiser-Kupfer MI. Complications of cystinosis after renal failure. Pediatr Nephrol 1987;1:260-8.

3 Gahl WA, Dalakas MC, Charnas L, et al. Myopathy and cystine storage in muscles in a patient with nephropathic cystine storage in muscles in a patient with
cystinosis. NEngl $\mathcal{Y}$ Med 1988;319:1461-4.

4 Fivush B, Flick JA, Gahl WA. Pancreatic exocrine insuffi ciency in a patient with nephropathic cystinosis. $\mathcal{F}$ Pediat 1988;112:49-51.

5 Thoene JG, Oshima RG, Crawhall JC, Olson DL, Schneider A. Cystinosis: intracellular cystine depletion by aminothiols in vitro and in vivo. 7 Clin Invest 1976;58:180-9.

6 Gahl WA, Tietze F, Butler JDeB, Schulman JD. Cysteamine depletes cystinotic leucocyte granular fractions of cystine by the mechanism of disulphide exchange. Biochem $\mathcal{f} 1985$ 228:545-50.

7 Gahl WA, Reed GF, Thoene JG, et al. Cysteamine therapy for children with nephropathic cystinosis. N Engl f Med 1987; 316:971-7.

8 Thoene JG, Lemons R. Cystine depletion of cystinotic tissues by phosphocysteamine (WR638). F Pediatr 1980;96:1043-4 Smolin LA, Clark KF, Thoene JG, Gahl WA, Schneider JA Smolin LA, Clark KF, Thoene JG, Gahl WA, Schneider JA. phosphocysteamine in elevating plasma cysteamine concen tration and decreasing leukocyte free cystine in nephropathic tration and decreasing leukocyte free cys

cystinosis. Pediatr Res 1988;23:616-20.
10 Haycock GB, Al-Dahhan J, Mak R, Chantler C. Effect of indomethacin on clinical progress and renal function in cystinosis. Arch Dis Child 1982;57:934-9.

11 Smolin LA, Schneider JA. Measurement of total plasma cysteamine using high-performance liquid chromatography with electrochemical detection. Anal Biochem 1988;168: 374-9.

12 Greene AA, Jones JA, Harms E, et al. Lysosomal cystine storage in cystinosis and mucolipidosis type II. Pediatr Res 1985; 19:1170-4.

13 Lowry OH, Rosebrough NR, Farr AL, Randall RJ. Protein measurement with the folin phenol reagent. $\mathcal{F}$ Biol Chem 1951;193:265-75.

14 Clifton PM, Chang L, Mackinnon AM. Development of an automated Lowry protein assay for the Cobas-Bio centriautomated Lowry protein assay for the Cob

15 Thoene JG, Oshima RG, Ritchie DG, Schneider JA. Cystinotic fibroblasts accumulate cystine from intracellular protein degradation. Proc Natl Acad Sci USA 1977;74:4505-7.

16 Jonas AJ, Schneider JA. Plasma cysteamine concentrations in children treated for cystinosis. F Pediatr 1982;100:321-3. 